\title{
Injury incidence and workloads during congested schedules in football
}

\begin{abstract}
This study compared injury incidence and training loads between single and multi-match weeks, and seasons with and without congested scheduling. Measures of internal (sessionRating of Perceived Exertion $\mathrm{x}$ duration for training/match and \% maximal heart rate) and external load (total, low-, high-, and very high-intensity running distances) along with injury incidence rates were determined from 42 players over 3 seasons; including 1 without and 2 (season 2 and 3) with regular multi-match weeks. Within-player analyses compared $1(n=214)$ vs 2 -match $(\mathrm{n}=86)$ weeks $(>75 \mathrm{~min}$ in matches), whilst team data was compared between seasons. Total injury rates were increased during multi-match weeks $(\mathrm{p}=0.001)$, resulting from increased match and training injuries $(50.3,16.9 / 1000 \mathrm{~h})$. Between-season total injury rates were highest when congested scheduling was greatest in season $3(27.3 / 1000 \mathrm{~h})$ and season $2(22.7 / 1000 \mathrm{~h})$ vs season $1(14.1 / 1000 \mathrm{~h} ; \mathrm{p}=0.021)$. All external load measures were reduced in multi-match weeks $(\mathrm{p}<0.05)$. Furthermore, all internal and external training loads were lowest in seasons with congestion $(\mathrm{p}<0.05)$. In conclusion, increased injury rates in training and matches exist. Total loads remain comparable between single and multi-match weeks, though reduce in congested seasons. Whether injuries result from reduced recovery, increased match exposure or the discreet match external loads remain to be elucidated.
\end{abstract}

Keywords: Soccer, multi-match weeks, Training Load, Champions League, Injury risk.

\section{Introduction}

Players in professional football teams are often involved in multiple concurrent competitions, including national and continental matches - with extremes of up to 60-70 matches/season reported [1]. Such competitive situations result in weeks with multiple (i.e. 2-3) matches and thus truncated recovery periods. These occurrences are commonly referred to as congested schedules or multi-match weeks and describe prolonged periods where multiple matches are played with $<3$ or 4 days of recovery [2-4]. Currently, mixed findings exist on the likelihood of injury or reduced performance during congested scheduling $[2,4,5]$, though a growing number of studies suggest increased injury rates during times of fixture congestion when compared to normal scheduling [2, 6, 7]. The growing eminence of the Asian Football Confederation (AFC) Champions League competition, combined with small squad sizes and limited budgets in Australian teams, has increased the concern over congested scheduling in the Australian domestic competition, though evidence of its impact remains sparse.

Research on injury rates during congested schedules remains equivocal. Although total injury count does not differ with respect to shorter or longer between-match recovery times [3], match and training injury rates (based on exposure time) are reported to increase, along with an increase in the severity of injuries sustained [3, 5]. For example, Bengtsson et al. [6] reported increased injury rates in league matches with 4 days compared to 6 days recovery (29.0 v 26.6 /1000h, respectively; $p=0.045)$. Further, Dupont et al. [5] reported an increase of 
4.1 to 25.6 injuries/1000 hours $(\mathrm{p}<0.01)$ in matches during non-congested and congested periods ( $<4$ days) in the Scottish Premier League. Recently, Bengtsson et al. [2] also reported that injury rates increase by $21 \%$ when $<3$ days separate matches in comparison to matches separated by $>6$ days in a sample of over 45,000 observations [2]. Conversely, Carling et al. [8] reported no difference to injury rates $(50.3$ vs $49.8 / 1000 \mathrm{~h} ; \mathrm{n}=19)$ for French professional players' in a congested period of 8 matches in 26 days $(\mathrm{p}=0.94)$. These different findings may result from methodological issues relating to varying sample sizes used (ie $n=8-32$ players) and larger squad sizes/depth available in particular European football clubs allowing for increased player rotation, all of which may obscure injury outcomes.

Despite equivocality existing for injury rates in congested scheduling, the training and match loads that precede injury during such periods remain unknown. The physical work performed during training and matches is commonly referred to as external load, while the psychophysiological response is considered internal load [9]. An understanding of external and internal load during congested periods is important given the relationship that is proposed to exist between load and injury [9]. Research to date reports no significant differences in distances covered in matches during or outside of congested schedules $[5,7,8]$. Further, Carling et al. [8] concluded that players are able to maintain movement patterns, particularly high-intensity efforts, even with short recovery ( $<3$ days) between matches. Despite these inmatch descriptions of fixture congestion, as yet training load distribution during these periods remains to be reported. The description of internal and external loads throughout congested periods may be beneficial to give context to injury incidences given the load-injury relationships reported recently [10-12], alongside training exposure needed to maintain fitness [13]. Understanding training load distributions gives further context to the loads encountered during congested schedules $[4,14]$ and may provide insight to the underlying reasons for mixed findings on injury rates outlined earlier [5, 11, 12].

The purpose for the present study is to examine the injury rates sustained during single match vs multi-match weeks and between seasons with and without congested schedules for a professional Australian club competing in domestic and AFC Champions League competitions. Further, an additional aim was to compare the respective training and match loads in SM and MM weeks and between seasons with and without congested schedules. It was hypothesized that MM weeks would exhibit higher injury rates than SM weeks, despite a reduction in training session loads.

\section{Methods}

\section{Participants}

The current case study prospectively examined one professional football team competing over 3 seasons in the highest competitive level in Australia (A-League). During seasons 2 and 3 the team also concurrently competed in the AFC Champions League, which consequently resulted in an increase in MM weeks. Data was collected from a total of 42 contracted players during this time with data included for 28 who competed in MM weeks, excluding goal keepers and those without match time. The players had a mean \pm SD age of $26.4 \pm 5.1 \mathrm{y}$, stature of $181.3 \pm 7.1 \mathrm{~cm}$, and body mass of $74.5 \pm 12.1 \mathrm{~kg}$. During periods of data collection, players were participating in 3-5 football-specific field-based training sessions, 1-2 gym/recovery sessions, and 1-2 competitive matches per week. All players volunteered to participate and prior to the commencement of the study, were informed of any risk associated with their involvement and provided consent before being included. The study was approved by the institutional Human Research Ethics Committee (2014000355). Further, the study generally meets the ethical standards cited for the International Journal Sports Medicine [15]. 


\section{Overview}

Data was collected from a total of 514 training sessions and 106 matches over three A-League seasons in 2012-2015 (pre-season and competition). The latter two seasons also included AFC Champions League matches ( $n=37)$, leading to regular MM weeks $(n=40)$ from both seasons. A limitation of the current study was the playing group that participated over the three seasons, which included 10 players completing all three seasons, 5 completed two consecutive seasons (2013-15), while 13 only competed in 1 season (2012-13). Given the multiple definitions used, herein SM weeks include matches separated by $>6$ days, whilst MM weeks were separated by $<4$ days within a 'typical' week of Monday-Sunday micro-cycle [5]. Data was only included from players who completed $>75$ min of match time in the SM and within both matches of the MM weeks to allow for direct comparison. Consequently, 214 and 86 data points were collected from players completing SM and MM weeks respectively. Further, across each season, MM weeks only occurred in seasons 2 and 3, with 18 and 22 congested schedule matches played in each season respectively. For within-season analyses, typically weekly micro-cycles for SM weeks included a weekend match with 4 training days of varying intensity; where MM weeks included 2 matches and 2-3 training sessions. External and internal markers of load were collected from all training days, except recovery/travel days or recovery days where only wellness was collected. For between-season analyses, all players who participated in matches from season $1(n=19), 2(n=22)$ and $3(n=31)$ were included with their training and match data pooled as means and used for analyses.

Markers of internal load, external load and injuries were collected from players each session within each season. However, data from global positioning systems (GPS) was not collected during matches due to Football Internationale de Federation Association (FIFA) regulations. As such, comparisons between external markers of load between SM and MM weeks are from training only. Further, in season 1 insufficient GPS units were available and therefore comparisons in external load between seasons were not performed. The researchers acknowledge these limitations of the research.

\section{Internal Load}

Respective training and match loads, reported as arbitrary units (AU), were calculated by multiplying each players training or match duration (min) by their session rating of perceived exertion (sRPE) recorded approximately 30 min following each session [14]. Total loads were calculated as the sum of training (practice) and match load and reported as a mean and total weekly and season load. Training load was also calculated with sRPE post session but solely based on 'practice time' and reported as session and weekly training load. Heart rate (HR) was collected during training (T31, Polar Electro, Kempele, Finland) from all players and reported as a percentage of maximal heart rate (\%MaxHR) and time greater than 85 percent (HR85\%) as per Krustrup et al., [16]. Maximal heart rate for each player was obtained from pre-season $\mathrm{VO}_{2 \max }$ testing using an incremental treadmill test, though not reported here. The testing procedure required players to run at $12 \mathrm{~km} / \mathrm{h}$ while the treadmill increased $1.5 \%$ each minute until volitional exhaustion. The test was terminated when the participant voluntarily stopped due to fatigue. All erroneous and missing HR data $(<3 \%)$ was removed from the data set prior to analysis.

\section{External Load}

During each training session, total distance $(\mathrm{m})$, mean speed $(\mathrm{m} / \mathrm{min})$ and the distance covered $(\mathrm{m})$ in three pre-defined categories which are commonly used within professional football $[17,18]$; low-intensity activity (LIR) $\left(<14.4 \mathrm{~km} \cdot \mathrm{h}^{-1}\right)$; high-intensity running (HIR) $(>14.5$ 
$\left.\mathrm{km}^{\mathrm{h}} \mathrm{h}^{-1}\right)$; and very-high intensity running (VHIR) $\left(>20 \mathrm{~km} \cdot \mathrm{h}^{-1}\right)$ were measured via $15-\mathrm{Hz}$ Global Positioning Satellite (GPS) devices (SPI HPU GPSports, Canberra, Australia). For each training session, players wore the same individually assigned device to reduce interdevice reliability issues. Devices were worn between the scapulae in a customized harness and data was subsequently analyzed using device specific software (Team AMS, GPSports, Canberra, Australia). The GPS units in this study have been reported to have an acceptable level of accuracy and reliability for measures of total distance (interclass correlation [ICC], $\mathrm{r}<0.53$; coefficient of variation [CV] 5-15\%) [19]. In contrast to the previous research, GPS units have reduced reliability when measuring very high intensity movements [20], with differences ranging from $5.68 \%$ to $9.81 \%$ (CV) for all speed measures [21]. Additionally, a $100 \mathrm{~Hz}$ accelerometer with a $16 \mathrm{G}$ tri-axis exists within the unit and is used to calculate body $\operatorname{load}^{\mathrm{TM}}$ (AU) by summing movement in all three planes of motion [20].

\section{Injury}

The club physiotherapist recorded all injuries in consultation with medical and conditioning staff. The same club doctor was present for the duration of the study whilst two different physiotherapists were employed by the club, with one physiotherapist completing the whole of season 1 and other completing both season 2 and 3 - though both were trained and complied with an injury reporting system part of the National Federation and Orchard Sports Injury Classification System (OSICS) requirements. An injury was defined as 'any physical complaint sustained from a match or training session which resulted in a partially completed session. Further, any injury sustained previously from training or match and resulting in unavailability for subsequent training and match' [22] was also deemed as an injury as dictated by the governing national body. Injury rates per 1000 hours (for training and matches respectively) were calculated as per other research [7]. Injury rates were calculated as a group mean for the match context (SM vs MM weeks) and squad means for comparison between seasons, which is similar to other research $[3,7,8]$ that has used squad injury rates ie. total injuries for the squad and mean exposure duration. Contact injuries were defined as an injury that was direct result of impact (either opposition or teammate), which included both muscular tissue and structural injuries. Non-contact injuries were defined as injuries without any impedance from another object, while match and training injuries were recorded based on which session type they occurred [3]. Finally, an injury that was sustained on match day or in the 4 days following was assigned to SM or MM week group accordingly [3].

\section{Statistical Analysis}

Data are presented as a mean \pm standard deviation (SD). For within-season comparisons, respective one-way repeated measures ANOVA was performed on log transformed data to determine differences in all load variables between SM and MM weeks. Separately, comparison between seasons for all load variables was via one-way repeated measures ANOVA. Statistical significance was set at $\mathrm{p}<0.05$ and post-hoc tests (Tukey) were used to determine differences between seasons. The Statistical Package for Social Sciences (SPSS v22.0, Chicago, IL) was used to perform all statistical analyses. Injury rates (/1000h) and injury counts were used to calculate an Incidence Rate Ratio (IRR) to determine whether there was higher injury risk in SM or MM weeks for within-season analyses and again for betweenseason analyses. Finally, 95\% confidence intervals (CIs) were calculated using z-statistics and ensuing $\mathrm{p}$ values [7].

\section{Results}

The mean number of training sessions per week and total session duration were significantly higher in SM weeks ( $\mathrm{p}=0.0001$; Table 1), despite session duration not being significantly 
different between SM and MM weeks ( $\mathrm{p}=0.77$; Table 1). All measures of internal training load (per session and weekly) were significantly greater during SM weeks ( $\mathrm{p}=0.001$; Table 1). However, total load per week was not significantly different between conditions $(\mathrm{p}=0.18$; Table 1). Training-based HR responses indicated \% max HR ( $\mathrm{p}=0.002)$ and HR85\% max $(\mathrm{p}=0.0001$; Table 1) were both higher for SM weeks. For external load markers, session and weekly total distance and mean speeds were significantly greater in SM than MM weeks $(\mathrm{p}=0.005$; Table 1). Total distance and distance within speed zones were lower in MM weeks, with lower session LIR, HIR and VHIR ( $\mathrm{p}=0.001$; Table 1$)$.

Comparison of SM vs MM total injury rates (relative to training and match duration) showed significantly greater injury rates during MM weeks $(\mathrm{p}=0.001$; Table 2$)$. Consequently, there was also a higher risk of total injury in MM weeks (IRR: 2.16 [95\% CI 1.2 - 5.6]; p<0.05). Furthermore, training (IRR: 2.52 [95\% CI 1.3 - 10.2]; $\mathrm{p}<0.05$ ) and match related injuries (IRR: 1.12 [95\% CI 1.1 - 3.6]; $\mathrm{p}<0.05$ ) were higher during MM weeks for both risk and incidence.

For between-season analyses, the number of training sessions completed in season 3 was significantly lower than season $1(\mathrm{p}=0.002$; Table 3$)$ and $2(\mathrm{p}=0.0001$; Table 3$)$. Weekly session duration was reduced across each season, with season 1 significantly higher than both season 2 and 3 ( $\mathrm{p}=0.001, \mathrm{p}=0.002$ respectively; Table 3 ), while season 2 was significantly higher than season $3(\mathrm{p}=0.001$; Table 3$)$. Internal training loads were significantly reduced in season 3 and 2 compared to $1(\mathrm{p}=0.005$; Table 3 ). Total weekly load was significantly lower in both seasons 3 and 2 when compared to season 1 , respectively ( $\mathrm{p}=0.003, \mathrm{p}=0.003$; Table 3 ); though not significantly different between seasons 2 and 3 ( $p=0.12$; Table 3 ). Regardless of congested scheduling, match loads and durations did not significantly differ between seasons $(\mathrm{p}=0.09$; Table 3$)$.

Significant differences existed between seasons 1 and 2 for total (IRR: 2.13 (95\% CI 1.3 - 3.9, $\mathrm{p}<0.05$ ), match (IRR: 2.67 [95\% CI $1.1-3.3$ ] ; $\mathrm{p}<0.05$ ) and training injury risk (IRR: 1.61 [95\% CI 1.2 - 4.8]; $\mathrm{p}<0.05$ ). Similarly, significant differences were also evident between seasons 1 and 3 for total (IRR: 2.58 (95\% CI 1.9 - 14.2, p<0.05), match (IRR: 2.76 [95\% CI $1.3-6.2] \mathrm{p}<0.05$ ) and training injury risk (IRR: 2.05 [95\% CI $1.6-11.3$ ]; $\mathrm{p}<0.05$ ). Significant differences also existed between season 2 and 3 for total, match and training injury rates ( $\mathrm{p}<0.05$; Table 2). Comparisons with IRR analyses showed a significant difference for total (IRR: 1.89 [95\% CI 1.4 - 11.2]; p<0.05), match (IRR: 1.92 [95\% CI 1.4 - 11.2]; $p<0.05$ ) and training injury risk (IRR: 1.72 [95\% CI 1.3 - 4.7]; $p>0.05$ ) between seasons 2 and 3.

\section{Discussion}

The current study represents a novel examination of the influence of fixture congestion within and between seasons on training loads and injury in football, within the context of the AFC Champions League. The main findings were that injury rates were highest during MM weeks, which was evident on direct comparison between SM vs MM weeks and between seasons with and without congested scheduling demands. Not surprisingly, match loads were increased during MM weeks, with increased match exposures potentially the main driver for increased injury rates observed. Total loads were not significantly different between SM v MM weeks, despite training loads being lower in MM weeks. Similarly, external training load measures were also reduced in MM weeks, and reduced in seasons with more congested fixtures. Accordingly, the nature of congested schedules results in reduced training loads, increased match demands, potential reduced recovery and concomitantly results in increased injury occurrence. The nature of this injury response may in turn relate to the explicit 
exposure to match loads, the discreet nature of external match loads or lack of appropriate recovery between matches.

\section{Injury in congested and non-congested schedules}

Previous research reports increased injury rates in professional UEFA football players in 2 vs 1 week matches (IRR: 2.0 (95\% CI: 1.1 to 3.8) when competing in concurrent domestic and Champions League competitions [3, 5, 7). Further, research [2] investigating match injury rates within UEFA competitions found that injury rates increased by around $20 \%$ in matches played with $<3$ days vs $>6$ days of recovery [2]. Despite these findings, evidence from outside of European football is lacking, and thus these findings from an AFC Champions League context provide agreement, suggesting increased injury rates during MM vs SM weeks. As a further explanation of increased injury rates, injuries increased due to match-based, noncontact injuries; which again concurs with previous observations in multi-match UEFA Champions League football injuries [5]. However, in contrast to the aforementioned research, training injury rates in the current study were significantly increased during MM weeks in comparison to SM $[3,5]$ despite significant reductions in training load. The training injury rate observed in the current study $(16.9 / 1000 \mathrm{~h})$ is higher than those previously reported for 2 match weeks in professional Scottish football $(8.3 / 1000 \mathrm{~h})$, and from congested periods in France (4.6/1000h). These increases may be exacerbated as a result of the unique demands imposed on Australian teams competing in domestic and Champions League competitions, whereby small squad sizes and greater travel demands may impede recovery during times of increased fixture congestion.

Whilst previous studies report increased injury rates in multi-match weeks from pooled team data sets in congested periods $[2,3,5,8]$, this is the first study to report increased injury rates in seasons with and without congested scheduling; though obvious limitations exist regarding different playing squads, these results are interpreted alongside the SM vs MM results previously discussed. An increased injury rate, particularly from increased match-based injuries was present in seasons with regular fixture congestion. Such a finding concurs with the findings of match-based injuries in a team competing in the UEFA Champions League [23]. However, the injury rates in particular seasons reported in the current study are lower than previous research; likely due to the playing squad in the current study being smaller than those available in Europe [4]. Regardless, a novel finding of the present study showed significant increases of training-based injuries during multi-match seasons, particularly noncontact muscular injuries that is consistent with the outcome of pooled $1 \mathrm{vs}$ match week data from within-player analyses.

\section{Training loads in congested and non-congested schedules}

Recently the influence of training loads as a precursor to injury occurrence have received growing research attention $[10,11]$. For example, despite recent conjecture, higher total training loads and the rate of increase in load are suggested to be related to higher overall injury incidence in players of various football codes [10, 24]. In a congested schedule context, coaches will intuitively adjust training loads during congested schedules, though changes to this workload profile given the knowledge of the training load - injury paradigm remains to be reported. Accordingly, total loads reported in the current study did not differ; however the reductions in training load that occurred in MM weeks were offset by the increased match load. Interestingly however, weekly training and total loads (including match load) were both significantly reduced in seasons with increased congested scheduling, with the lowest loads reported in season 3 inclusive of the highest number of matches. Previous research [13] on sub-elite university footballers has shown reduced training loads result from MM weeks and 
in turn directly reduces physical capacity over a 6 week period. Furthermore, high match loads with regular MM weeks (ie. $3^{+}$matches with $<4$ days recovery) appear to increase the risk of accumulative fatigue in both sub-elite and professional football players $[13,25]$. Recently, training load distribution in 2 and 3 match weeks reported a decrease in training duration as the frequency of matches within a week increases [26]. Alongside these findings, the current study provides evidence that in this case study of one team, no difference in total sRPE load was evident and loading in multiple matches is unlikely to be the cause of injury per se given the discreet time periods analysed here. However, increased match sRPE load and reduced recovery between matches may reduce player's ability to cope with the same total sRPE load and be an antecedent for injury occurrence, particularly during prolonged exposure to these demands and deserves further attention.

The majority of research on congested schedules relating to external load has focused on the physical demands during matches $[1,3,7,8,23]$. The current study represents a novel report of the distribution of external loads during training across multiple seasons with fixture congestion. External load measures were all significantly reduced during weeks with congested matches, although a limitation here is that external match demands are not reported in the current study, which is likely to be a factor in injury occurrence [27]. The total $(3717 \pm 797 \mathrm{~m})$ and high speed running distance $(436 \pm 192 \mathrm{~m})$ recorded in the current study for 1 match weeks is similar to those previously reported for English Premier League (EPL) players (e.g. daily total 3-4 km and high speed running distance (285-442m) [28]. Despite total distance in the current study for SM weeks being somewhat similar to those previously reported [18, 28], MM weeks had significant reductions for total distance, HIR and VHIR. Although the current study did not report external load for matches, the current findings show that training sessions are altered to accommodate for the increased match load of MM weeks. We also were unable to report GPS data for season 1, and thus the nuanced responses to increased external load in training for seasons with congested schedules (i.e Season 2 and 3 here), remains to be determined. Similarly, weekly total distances for EPL players supports the current research, reporting significantly less distance during training in weeks with multiple matches compared with SM weeks [26]. Accordingly, multi match weeks, which includes both 2 and 3 matches reported higher total, and zone running distances, and highlights a limitation of the lack of external load data of the present study.

\section{Conclusions}

The present study examined fixture congestion within and between seasons in Australian domestic and Champions League football to determine the effect on training loads and injury. The main findings showed that total training and match injury rates increased as a result of fixture congestion within and between seasons. Further, internal and external markers of load were also significantly reduced during congested weeks and seasons, though despite these reductions, the risk of total injury and significantly training based injuries was still increased during fixture congestion. Although measures of fitness and fatigue were not assessed as part of the current study, it would be of interest to observe whether the changes in external and internal load reported here had any corollary effect. Future research may be able to assess these relationships, which might also include a marker of external load during matches.

\section{Practical Applications}

- Injury prevalence during fixture congestion is increased within and between seasons.

- Rotation of players can be considered during increased fixture congestion dependent on squad quality and availability. 
- Quantifying match loads is important to assist understand athlete loading during congested periods.

\section{Disclosure Statement}

No potential conflict of interest was reported by the authors.

\section{References}

${ }^{1}$ Odetoyinbo K, Wooster B, Lane A. The effect of a Succession of Matches on the Activity Profiles of Professional Soccer Players. Science and football VI. London: Routledge. 105-8.

${ }^{2}$ Bengtsson H, Ekstrand J, Waldén M, Hägglund M. Muscle injury rate in professional football is higher in matches played within 5 days since the previous match: a 14-year prospective study with more than 130000 match observations. Br J Sports Med 2018; 52:1116-1122.

${ }^{3}$ Dellal A, Lago-Peñas C, Rey E, Chamari K, Orhant E. The effects of a congested fixture period on physical performance, technical activity and injury rate during matches in a professional soccer team. Br J Sports Med 2015; 49:390-394.

${ }^{4}$ Ekstrand J, Hägglund M, Waldén M. Injury incidences and injury patterns in professional football: the UEFA-injury study. Bri J Sports Med 2011; 45:553-558. 
${ }^{5}$ Dupont G, Nedelec M, McCall A, McCormack D, Berthoin S, Wisløff U. Effect of 2 soccer matches in a week on physical performance and injury rate. Am J Sports Med 2010; 38:17521758.

${ }^{6}$ Bengtsson H, Ekstrand J, Hägglund M. Muscle injury rates in professional football increase with fixture congestion: an 11-year follow-up of the UEFA Champions League injury study. Br J Sports Med 2013; 47:743-747.

8
${ }^{7}$ Carling C, Gregson W, McCall A, Moreira A, Wong del P, Bradley PS. Match Running Performance During Fixture Congestion in Elite Soccer: Research Issues and Future Directions. Sports Med 2015; 45:605-613.

${ }^{8}$ Carling C, Le Gall F, Dupont G. Are physical performance and injury risk in a professional soccer team in match-play affected over a prolonged period of fixture congestion? Int J Sports Med 2012; 33:36-42.

${ }^{9}$ Impellizzeri FM, Marcora SM, Coutts AJ. Internal and External Training Load: 15 Years On. Int J Sports Physiol Perform 2019; 2:270-273.

${ }^{10}$ Gabbett JT, Jenkins GD. Relationship between training load and injury in professional rugby league players. J Sci Med Sport 2011; 14:204-206.

${ }^{11}$ Gabbett JT, Ullah S. Relationship between running loads and soft-tissue injury in elite team sport athletes. J Strength Cond Res 2012; 26:953-958.

${ }^{12}$ Rogalski B, Dawson B, Heasman J, Gabbett TJ. Training and game loads and injury risk in elite Australian footballers. J Sci Med Sport 2013; 16:499-503.

${ }^{13}$ Rollo I, Impellizzeri MF, Zago M, Iaia MF. Effect of 1 versus 2 games a week on physical and subjective scores of subelite soccer players. Int J Sports Phys 2014; 9:425-431.

${ }^{14}$ Foster C. Monitoring training in athletes with reference to overtraining syndrome. Med Sci Sports Exerc 1998; 30:1164-1168.

${ }^{15}$ Harriss DJ, Macsween A, Atkinson G. Standards for Ethics in Sport and Exercise Science Research: 2018 Update. Int J Sports Med 2017; 38:1126-1131.

${ }^{16}$ Krustrup P, Mohr M, Amstrup T, Rysgaard, T, Johansen J, Steensberg A, Pedersen PK, Bangsbo J. The Yo-Yo Intermittent Recovery Test: Physiological Response, Reliability, and Validity. Med Sci Sport Exercise 2003; 35;697-705.

${ }^{17}$ Coutts AJ, Duffield R. Validity and reliability of GPS devices for measuring movement demands of team sports. J Sci Med 2010; 13:133-135.

${ }^{18}$ Malone JJ, Di Michele R, Morgans R, Burgess D, Morton JP, Drust B. Seasonal Training Load Quantification in Elite English Premier League Soccer Players. Int J Sports Phys Per 2015; 10:489-497. 
${ }^{19}$ Vickery MW, Dascombe JB, Baker DJ, Higham GD, Spratford AW, Duffield R. Accuracy and Reliability of GPS Devices for Measurement of Sport Specific Movement Patterns related to Cricket, Tennis, and Field Based Team Sports. J Strength Cond Res 2014; 28:1697-1705.

${ }^{20}$ Johnston RJ, Watsford ML, Kelly SJ, Pine MJ, Spurrs RW. Validity and interunit reliability of $10 \mathrm{~Hz}$ and $15 \mathrm{~Hz}$ GPS units for assessing athlete movement demands. J Strength Cond Res 2014; 28:1649-1655.

${ }^{21}$ Waldron M, Worsfold P, Twist C, Lamb K. Concurrent validity and test-retest reliability of a global positioning system (GPS) and timing gates to assess sprint performance variables. J

11 Sports Sciences 2011; 29;1613-1619.

${ }^{22}$ Fuller CW, Ekstrand J, Junge A. Consensus statement on injury definitions and data collection procedures in studies of football (soccer) injuries. Scandinavian Journal Medicine Science Sports 2006

16 ; 40:83-92.

${ }^{23}$ Lago-Peñas C, Rey E, Lago-Ballesteros J, Casáis L, Domínguez E. The influence of a congested calendar on physical performance in elite soccer 2011; J Strength Cong Res. 25:2111-2117.

${ }^{24}$ Gabbett JT. The training-injury prevention paradox: should athletes be training smarter and harder? Br J Sports Med 2016; doi:10.113 6/bjsports-2015-095788.

${ }^{25}$ Beijsterveldt MA, Stubbe HJ, Schmikli LS, Van De Port IGL, Backx GDG. Differences in injury risk and characteristics between Dutch amateur and professional soccer players. J Sci Med Sport 2015; 18:145-149.

${ }^{26}$ Anderson L, Orme P, Michele DR, Close LG, Morgans R, Drust B, Morton PJ.

30 Quantification of training load during one-, two- and three-game week schedules in professional soccer players from the English Premier League: implications for carbohydrate periodisation. J Sports Sci 2016; 34:1250-1259.

${ }^{27}$ Carling C, McCall A, Le Gall F, Dupont G. The impact of short periods of match congestion on injury risk and patterns in an elite football club. Br J Sports Med 2015; 49:764768. Power Data. Int J Sports Med 2013; 34:963-968. 